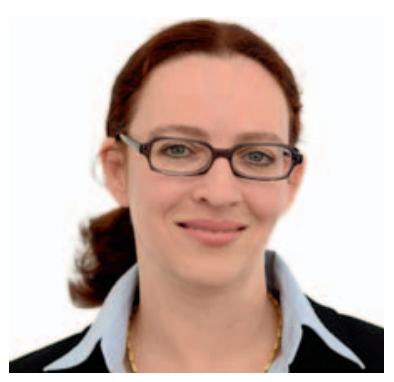

\title{
Onkologen sollten über die Misteltherapie informiert sein
}

\author{
Claudia Witt \\ Institut für komplementäre und integrative Medizin, UniversitätsSpital Zürich, Zürich, Schweiz
}

Abstractübersetzung aus Gschwendtner KM, Holmberg C, Weis J: Beweggründe von Krebspatienten für und gegen die Inanspruchnahme der Misteltherapie. Forsch Komplementmed 2016;23;215-222.

Beweggründe von Krebspatienten für und gegen die Inanspruchnahme der Misteltherapie

\section{Schlüsselwörter}

Misteltherapie · Komplementärmedizin · Krebs

Qualitative Studie · Patientenbedürfnisse

\section{Zusammenfassung}

Einleitung: Die Misteltherapie ist im deutschsprachigen Raum ein häufig angewandtes komplementärmedizinisches Verfahren (KM) in der Onkologie. Diese Studie hatte das Ziel, die Beweggründe für oder gegen eine Inanspruchnahme der Misteltherapie zu untersuchen und Themenfeldern zuzuordnen.

Patienten und Methoden: Es wurden qualitative leitfadengestützte Interviews mit Krebspatienten geführt. Der Interviewleitfaden fragte nach der Inanspruchnahme von KM, der Motivation zur Inanspruchnahme, Informationsverhalten und -bedürf- nissen zu KM sowie nach der Krebserkrankung. Um die Beweggründe für die Inanspruchnahme oder Nichtinanspruchnahme der Misteltherapie zu verstehen, wurden die Interviews inhaltsanalytisch ausgewertet.

Ergebnisse: Insgesamt wurden Interviews mit 88 Krebspatienten geführt, davon nutzen 18 (20,5\%) die Misteltherapie. Die Beweggründe für oder gegen eine Inanspruchnahme der Misteltherapie ließen sich den 2 Themenfeldern «Wahrgenommene Indikation» und «Abwägungen bei der Entscheidungsfindung» zuordnen.

Diskussion und Schlussfolgerungen: Mit der Misteltherapie wird sowohl ein Einfluss auf das Tumorwachstum als auch eine supportive Wirkung assoziiert. Anwender sehen die Misteltherapie als sicheres Verfahren; Nichtnutzer befürchten eher Nebenoder Wechselwirkungen. Die Empfehlung von Fachpersonal spielt eine wichtige Rolle bei der Inanspruchnahme. Zum Teil waren die Nichtnutzer interessiert an der Anwendung der Misteltherapie, befanden sich jedoch noch im Klärungsprozess.

(c) 2017 S. Karger GmbH, Freiburg

\section{KARGER}

Fax +497614520714 information@karger.com www.karger.com

\section{(C) 2017 S. Karger GmbH, Freiburg}




\section{Transfer in die Praxis}

\section{Hintergrund}

Die Misteltherapie ist insbesondere im deutschsprachigen Raum weit verbreitet und nimmt eine besondere Rolle in der komplementärmedizinischen Behandlung von Krebspatienten ein. Die meisten Onkologen und Patienten haben schon von der Misteltherapie gehört, ihr Nutzen wird jedoch oft kontrovers diskutiert.

\section{Studienergebnisse}

Gschwendtner et. al. haben mit ihrer Studie einen wichtigen Beitrag zum Verständnis der patientenseitigen Beweggründe für eine Inanspruchnahme der Misteltherapie geleistet. Die Studie wurde im Rahmen des Kompetenznetzes Komplementärmedizin in der Onkologie (KOKON) durchgeführt, das von der Deutschen Krebshilfe gefördert wurde. Es handelt sich um eine qualitative Studie, die vom Design her gut geeignet ist, um Fragen nach dem «Wie» und «Warum» zu beantworten, und damit auch hilft, Hypothesen für quantitative Studien abzuleiten. Angesichts der 88 Patienteninterviews wurde eine für diesen Studientyp große Anzahl von Patienten eingeschlossen. Von den Interviewten nahm etwa ein Fünftel eine Misteltherapie in Anspruch. Die Ergebnisse zeigen neben einer kontroversen Beurteilung der Wirkung der Misteltherapie auch die unterschiedliche Einschätzung von Nutzern und Nichtnutzern bezüglich der Sicherheit der Therapie. Ob es sich aus Sicht der Patienten um ein sicheres Verfahren handelt, hatte einen relevanten Einfluss auf die Entscheidung für oder gegen eine solche Therapie. Bei Nichtnutzern wurden unter anderem mögliche Nebenwirkungen, Interaktionen mit anderen Medikamenten und das mögliche Auftreten von Allergien als Grund der Nichtanwendung angeführt.
Es wurde auch deutlich, dass eine Empfehlung durch das Fachpersonal für die meisten Patienten ein wichtiger Aspekt bei der Entscheidung war.

\section{Fazit für die Praxis}

Die Misteltherapie ist im deutschsprachigen Raum weit verbreitet, und es ist wichtig, dass onkologisch behandelnde Ärztinnen und Ärzte über den möglichen Nutzen und potentielle Nebenwirkungen sowie Interaktionen informiert sind, da ihre Empfehlungen einen relevanten Einfluss auf die Entscheidung ihrer Patienten haben. Für interessierte Ärztinnen und Ärzte findet sich auf Onkopedia [1] eine Zusammenfassung zur Misteltherapie, die in Kooperation von KOKON und dem CAM-Cancer Consortium erstellt wurde.

\section{Disclosure Statement}

Hiermit erkläre ich, dass keine Interessenskonflikte in Bezug auf den vorliegenden Kommentar bestehen.

\section{Literatur}

1 Onkopedia: Mistel (Viscum album). www.onkopedia.com/de/onkopedial guidelines/mistel-viscum-album/@@view/html/index.html_(Zugriff 24.01.2017).

Kontaktadresse: Prof. Dr. Claudia Witt, Institut für komplementäre und integrative Medizin, UniversitätsSpital Zürich, Rämistrasse 1000, 8091 Zürich, Schweiz, claudia.witt@uzh.ch 